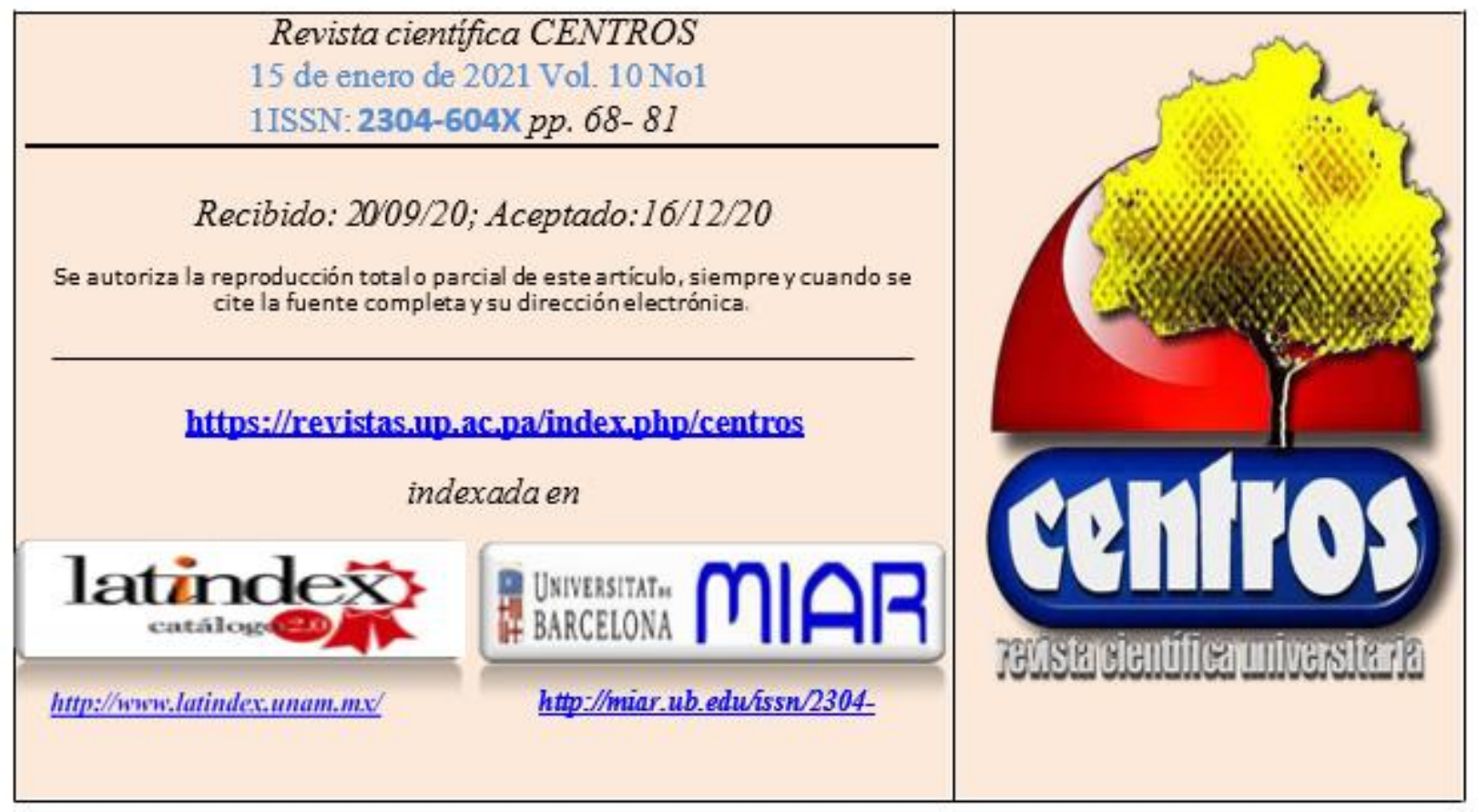

\title{
DESCENTRALIZACIÓN EDUCATIVA EN EL MUNICIPIO CERTIFICADO DE TUNJA: UN PROCESO COMPLEJO EN TIEMPO DE POSTCONFLICTO ${ }^{1}$
}

\author{
José Alberto Moreno Villamil \\ Universidad Pedagógica y Tecnológica de Colombia (UPTC) / \\ https://orcid.org/0000-0001-8761-8011 \\ joalmovil@gmail.com
}

\section{Resumen}

Este artículo plantea una reflexión sobre la complejidad de la descentralización educativa en el municipio Certificado de Tunja, enmarcada dentro de la investigación llevado a cabo en mi tesis doctoral titulada "Descentralización Educativa en el Municipio Certificado de Tunja, desde una visión del pensamiento complejo", donde los elementos

\footnotetext{
${ }^{1}$ Esta investigación se enmarca en el contexto del Proyecto de Investigación titulado "Descentralización Educativa en el Municipio Certificado de Tunja, desde una visión del pensamiento complejo", vinculado con las líneas de investigación del doctorado en Ciencias de la Educación de RUDECOLOMBIA de la Universidad Pedagógica y Tecnológica de Colombia (UPTC), específicamente la referida a Universidad y Nación, cuya intención se encuentra establecida en la línea específica Formación de Educadores e Interculturalidad.
} 
de calidad, cobertura y eficiencia, generan grandes expectativas e interrogantes sobre el verdadero impacto de las nuevas reglas de juego, que modificaron las relaciones de poder entre el Estado y las entidades territoriales, y, por ende estos últimos debían tener una mayor capacidad técnica y de gestión para manejar el sistema educativo y construir espacios reales de autonomía en la búsqueda de generar políticas educativas desde lo local, que respondan a la coyuntura actual frente al cumplimiento de los acuerdos de La Habana, en tiempos de transición donde el sector educativo tunjano no es ajeno a esta nueva realidad cambiante orientada a la inclusión y a la reconciliación.

Palabras clave: Complejidad, descentralización educativa, autonomía, inclusión y postconflicto.

\section{Abstract}

This article presents a reflection on the complexity of educational decentralization in the Certified Municipality of Tunja, framed within the research carried out in my doctoral thesis entitled "Educational Decentralization in the Certified Municipality of Tunja, from a perspective of complex thinking", where the elements of quality, coverage and efficiency generate great expectations and questions about the true impact of the new rules of the game, which modified the power relations between the State and territorial entities, and therefore the latter should have a greater technical and managerial capacity to manage the educational system and build real spaces of autonomy in the search to generate educational policies from the local level, that respond to the current situation regarding compliance with the Havana agreements, in times of transition where the sector educational tunjano is no stranger to this new changing reality ori initiates inclusion and reconciliation.

Keywords: complexity, educational decentralization, autonomy, inclusion and postconflict.

\section{Introducción}

Develar la naturaleza de la complejidad subyacente al proceso de descentralización educativa en el municipio certificado Tunja, pasa por estudiar las 
relaciones de poder en Colombia; relaciones que han estado latente entre el manejo central del poder y la descentralización o reparto del mismo, situación que se evidenció en las distintas constituciones y disposiciones normativas que desde la génesis de la arquitectura institucional nacional hasta nuestros días, se han establecido en el ordenamiento jurídico y han generado dinámicas particulares en el ámbito educativo.

Desde esta perspectiva, se considera que existen razones suficientes para reconocer importantes adelantos en el tema de investigación en cuanto a los ejercicios territoriales de construcción de políticas públicas para fortalecer la educación, sin embargo, se debe hacer el ejercicio de reflexión para evaluar la descentralización educativa en el Municipio de Tunja y la voluntad política del nivel central para profundizar una lectura del reparto de poder y orientar la disposición de los recursos financieros necesarios, para atender de mejor forma la educación en el municipio, que enfrenta grandes desafíos de inclusión en estos tiempos de postconflicto.

La nueva dimensión jurídica del estado social de derecho consagrado en la carta política de 1991, implica la génesis de un modelo de organización y funcionamiento del Estado, que parte de la adopción de un novedoso marco institucional, el cual desarrolla instrumentos conducentes a mejorar la eficiencia del suministro de servicios públicos y a aumentar la participación ciudadana en la toma de decisiones. Por lo que Sánchez refiere:

El proceso descentralizador en Colombia trata de abrir a las instituciones escenarios de integración y cooperación con organizaciones sociales, rompiendo la concentración de las relaciones entre el Estado y los gremios y las organizaciones del capital, en relación con los sindicatos y organizaciones de trabajo. Viéndose como una alternativa de modernizar el aparato institucional a nivel central y regional, planificación y asignación de recursos, para generar una autonomía departamental, de intendencias, comisarías y de los municipios, analizada desde una etapa inicial en el año de $1989^{2}$.

\footnotetext{
${ }^{2}$ Sánchez, A. (2016). Revisión histórica del proceso de descentralización administrativa y sus restos en Colombia, de 1985 a 2016. Trabajo de grado para optar por el título de Magister en Planeación para el Desarrollo. Universidad Santo Tomás. Colombia.
} 
Más no se trata tan sólo, de un mero escrito que establece las relaciones con todos los actores, sino que esta etapa representa el inicio evidente de nuevas relaciones de poder con la administración pública, ya no es sólo el sector hegemónico quien controla sino a través de la interacción de todos los actores quienes confluyen para la planeación y gestión de los recursos públicos.

Conviene subrayar entonces, que los procesos de descentralización son herramientas que brindan mayor equidad y eficiencia del Estado, con el objetivo de satisfacer las necesidades de los ciudadanos, con base en encuentros y diálogos, con todos los contextos tanto territorial como local; donde el ciudadano tiene el derecho a participar y sobre todo a tomar decisiones sobre lo que realmente son sus necesidades con autonomía y conocimiento, porque es de su propia realidad. Y de acuerdo con el planteamiento de Delgadillo 3 "la descentralización es un proceso integral para devolverle el Estado a las provincias colombianas mediante la entrega de los recursos fiscales, autonomía de planificación y autonomía política para asumir las responsabilidades regionales".

No obstante, conviene entender las transformaciones que han sucedido y han dado paso a los cambios en la forma de ser y relacionarse de los estados, que dio como resultado un Estado social, fundado sus bases en los espacios de contacto entre lo ciudadano y lo estatal, lo que Gómez Aranguren plantea:

Lo estatal resultó insuficiente para atender los requerimientos de los social, esto opera como un vector que presiona las transformaciones constitucionales que luego se estatuyen mediante las formas jurídicos políticas que en el fondo no son otra cosa que la definición jurídica de realidades ya advertidas ${ }^{4}$.

\footnotetext{
${ }^{3}$ Delgadillo, G. Descentralización administrativa, desconcentración y delegación de funciones. Tesis para optar al Título de Especialista en Derecho Público. Universidad Nacional de Colombia, Santa Fe de Bogotá, Colombia. 1989. $23 \mathrm{p}$.

${ }^{4}$ Gómez Aranguren, G. Principios del derecho Administrativo. ABC Editores, Librería Limitada. Bogotá. 2002. 76 p.
} 
La transformación del Estado intervencionista, trajo como consecuencia el surgimiento de la descentralización funcional, que en la práctica no era más que las atribuciones del Estado en el manejo en todo a lo que refería a la administración pública y de servicios públicos, en el colapso de competencias, que hizo necesaria la diversificación administrativa de las autoridades centrales, de manera que para atender los servicios debían descargar toda autoridad central a estructuras que operativamente pudiesen garantizar mayor eficiencia en la satisfacción de las necesidades sociales.

Se debe agregar que el Estado intervencionista no solo refiere a la prestación de servicios públicos sino a todas aquellas actividades que buscan el fomento y estimulo de acciones que constituyan un cometido público, cuya misión en la descentralización funcional era la creación de organizaciones que se encarguen de promover la dinamización de la economía en función del desarrollo social.

Hecha esta salvedad, se menciona otro mecanismo de la descentralización, conceptualizada como descentralización territorial, la cual trata básicamente de una necesidad local que debe ser atendida por la administración mediante una estructura organizacional que goce de autonomía administrativa, lo que reconoce mayor eficiencia en el cumplimiento de las funciones estatales. En razón a ello:

Es necesario destacar que el Estado centralizado, en lo administrativo otorga, razón de ser al fenómeno descentralista, cuyo contenido esencial se radica en que las atribuciones de la autoridad central, son análogos o concurrentes a los entes descentralizados, tan solo que por virtud de esta circunstancia geográficas, esas mismas funciones se trasladan a los territorios para garantizar como se anotaba, eficiencia, autonomía y en fin una mayor proximidad entre los ciudadanos y las estructuras administrativas del Estado ${ }^{5}$.

Deseo subrayar en este aparte, que la descentralización no sólo es un fenómeno

\footnotetext{
${ }^{5}$ Ibíd., p. 90.
} 
de traslado de competencia, sino de radicación de atribuciones distintas y por tanto complejas, ya que poseen naturaleza diferente, tanto del ente central como del ente territorial, bajo autoridades únicas, cuya cobertura solo es posible desde el ámbito procedimental de cada una. En caso contrario, si esto en su forma no sucediese, se trataría solo de un reparto de responsabilidades y atribuciones públicas entre los diversos entes del Estado. Conforme a ello, la descentralización territorial coexiste con entidades de competencia propia, como por ejemplo el departamento y el municipio, ambos pueden llegar ser afines en la prestación de servicios público, en el caso de la educación, de allí, el principio de complementariedad que Morin ${ }^{6}$ plantea, y establecido en la constitución política como característica básica de funcionamiento de los entes territoriales.

Dicha complejidad llevada al espacio territorial de Tunja y a la dinámica del sistema educativo en clave de posturas incluyentes con diferentes sectores de la población y particularmente con jóvenes cuyas familias estuvieron afectadas por el conflicto que vivió el país durante más de diez lustros, se observa la inserción de 432 niños en las instituciones oficiales de Tunja en educación inicial básica y media durante el año lectivo de 2019. Esta situación muestra el reto del sistema educativo tunjano de aportar a la reconciliación nacional y ofrecer amplia cobertura y unas trayectorias educativas completas para esa población que vivió en sus entornos familiares la tragedia de la guerra y que quiere rehacer su vida bajo nuevos modelos de convivencia esta capital.

La pretensión de esta reflexión, es hacer notar que el método que se propone para abordar la investigación a través de una realidad empírica, parte de las visiones de la complejidad; el pensamiento complejo sus principios y operadores lógicos; fundamentalmente en: el dialógico, la recursividad organizacional y el hologramático como procesos básicos, por lo que, de manera crítica y en cierto sentido, permitirá dar apertura a la dinámica del propio sistema (Morin, 1988; 1993; 1999a; 1999b; 2003; 2004).

De allí que se busca dejar por sentado, que el proceso de descentralización educativa y la inclusión de niños cuyas familias vivieron el conflicto, en el municipio

\footnotetext{
${ }^{6}$ Edgar Morin. El método II. La vida de la vida. Cátedra. (1993)
} 
certificado de Tunja, se observa como una realidad multidimensional, abierta, dinámica y compleja y no con una visión estática y fragmentada de la misma; por tanto, nuestra postura paradigmática se asienta en la ciencia de la complejidad, desde la visión moriniana del pensamiento complejo.

\section{Roles de la educación en tiempos de transición}

La visión compleja del proceso de descentralización educativa vislumbra el accionar del proceso de planificación de políticas como sistema dialógico individuoespecie-sociedad, siendo el eje central la ética, como elemento de desarrollo humano. Se desprende de allí, el propósito de transformación de la sociedad, orientado en gran medida a la satisfacción de las necesidades humanas para su desarrollo, lo cual exige un nuevo modo de comprender y explicar la realidad, y obliga a evaluar el mundo, las personas y sus procesos, de una manera distinta a la convencional.

Se postula que, a través de las líneas de trabajo del pensamiento complejo transdisciplinario, se puede ejecutar la visión en redes de una nueva conceptualización, de manera que cada una de las etapas del proceso, funcione de manera interconectada con el accionar estratégico, y sus resultados, se orienten hacia una estrategia integral, que incluya la mejora de la calidad de vida de la población, una educación basada en valores de convivencia pacífica y el respeto a la ley, para fortalecer la cohesión social y desarrollar una cultura de tolerancia y paz.

Desde esta visión, la gestión pública ejecutada por los diferentes elementos que conforman el Estado, brinden el soporte o sustrato necesario para facilitar la interacción, negociación, cooperación o resolución de conflictos entre los individuos, grupos u organizaciones, sujetos a la formulación de toda política educativa y de paz, en concordancia con una visión integral y continua de planificación, que regula su comportamiento a través de un marco normativo y administrativo eficiente y transparente.

Todo esto confirma, que Colombia atraviesa por un momento difícil en su devenir histórico, aunque se firmó la paz y se habla mucho de construir un camino de 
reconciliación; las muertes de numerosos líderes sociales, la falta de reparación a las víctimas, la no entrega de los bienes de la antigua insurgencia y varias dudas de la comunidad internacional sobre el cumplimiento de lo acordado, han generado diversos interrogantes sobre las nuevas relaciones de poder entre el estado y los actores sociales; así como a los espacios territoriales que enfrentan nuevas dinámicas en sus tejidos sociales.

Luego de tres largos años de la firma e implementación del prenombrado acuerdo de paz y ante los reparos actuales de incumplimiento por parte de las antiguos líderes de las extintas Farc, por la muerte de varios de sus integrantes y los señalamientos de indiferencia del Gobierno ante los temas pactados; es el tiempo de las salidas concertadas, de las miradas esperanzadoras, los consensos y las posturas incluyentes frente al cambio real y la posibilidad de construir colectivamente una nueva patria cuya pilar esencial es la educación como eje central del desarrollo.

En ese contexto nacional señalado en las anteriores líneas, nos encontramos ante dos posibilidades para los miembros de la academia: una es apartarse de las discusiones y refugiarse en los claustros y espacios del saber y la otra, es intervenir como actores protagónicos y deliberar en forma decidida y con mirada trasformadora de los contextos que los circundan, es decir pasar de las formulaciones teóricas a las posturas pragmáticas propicias para los cambios reales.

Ante esas dos alternativas, considero que la segunda vía es la más indicada, y en ese sentido le asiste razón a Rettberg cuando acertadamente plantea "por consiguiente, en tiempos de polarización social, propios de momentos de transición como el que atraviesa Colombia, la academia debe tener una vocación firme por el análisis sereno de los argumentos y de los hechos. Su rol no es ser neutral; no podría serlo. Su rol si debe ser el responder a la polarización con el respeto por la diferencia, y a la especulación con la cordura que resulta de un análisis crítico y honesto de la evidencia"7.

\footnotetext{
${ }^{7}$ Angélika Rettberg. Academia y reconciliación, en Reconciliación el gran desafío de Colombia 2015, Nomos impresores, 292
} 
En estos tiempos cambiantes, las distintas demandas sociales y la voluntad de los múltiples actores encargados de diseñar y precisar las estructuras organizacionales establecidas en una colectividad, en el marco de un contrato o pacto social fundado en determinados ámbitos normativos, inciden sustancialmente en el modelo o tipo de Estado instituido y asentado en un territorio.

Los nuevos modelos sociales de concordia en la sociedad colombiana inician en el seno de las instituciones educativas y van más allá del cumplimiento formal de unos manuales de convivencia y la observancia meramente teórica de las cátedras de la paz. Tienen que ver con compromisos reales donde la humanización del sistema educativo, sea la premisa mayor de los agentes formativos. No caben los casos de segregación o exclusión para los niños que llegan de otros lugares de la geografía nacional buscando nuevos horizontes de paz.

\section{Sistema educativo de Tunja en el posconflicto}

El municipio Tunja, es certificado a través de Resolución Ministerial No 2755 del 03 de diciembre de 2002, conforme a lo establecido en la Ley 715 de 2001, bajo una visión de Estado con permanentes procesos de transformación social, política y económica y con ello poder dar respuesta a reacciones, posturas y decisiones frente a las problemáticas que se presenten, por lo que le permitió al municipio iniciar de manera visible un proceso de consecución de la calidad educativa.

Bajo este principio de calidad educativa, el municipio certificado Tunja a nivel de gestión, ha alcanzado un buen uso de los recursos, que es preciso señalar son escasos, tratando de superar la visión individualista y fragmentada que ha predominado hasta la actualidad. En ese sentido, cabe destacar el valioso papel del sistema educativo de Tunja para que su territorio coadyuve en la construcción de un nuevo país. Es así como en el año 2019 se albergó a 432 niños cuyas familias tuvieron secuelas del conflicto armado y a 321 niños del hermano país de Venezuela que debieron dejar su nación por la crisis 
política y social que atraviesa. Es una manifestación clara de autonomía educativa de la ciudad de Tunja, que, ante la complejidad de su nuevo tejido social, permite la incorporación de estos estudiantes que enriquecen su cosmovisión y abren espacio a esquemas interculturales propios de miradas incluyentes que se requieren para construir nuevas sociedades.

No obstante, por Resolución Ministerial № 4278 del 20 de noviembre de 2019, se formulan cargos y se adopta de manera cautelar la Medida Correctiva de Suspensión de Giros de los recursos de la Participación de Educación del Sistema General de Participaciones correspondientes a la asignación para Calidad Matrícula Oficial del Municipio Tunja, Certificado en Educación; y se inicia la Actuación Administrativa de acuerdo con lo previsto por los artículos 208 del Estatuto Orgánico del Sistema Financiero y 14 del Decreto 028 de 2008 y sus normas reglamentarias.

Teniendo en cuenta que tal decisión gubernamental, conduce a un marcado proceso de deterioro de la calidad educativa, en cuanto a bienes, espacios y recursos didácticos, es muy compleja la situación que enfrenta el sistema educativo del municipio, tanto en el ámbito urbano como en el rural, llevando a una grave crisis que no contribuye a ampliar y fortalecer la democracia. Se limita fiscal, financiera, administrativa y económicamente, parte de la autonomía que ya habían alcanzado, el nivel nacional retoma muchas atribuciones, competencias y manejos, que reviven el asfixiante esquema centralista anterior a 1991. Por lo que presume una realidad contradictoria, donde el modelo actual, tiende más a la desconcentración que a la descentralización, entendida la primera como la cesión al nivel regional de algunas actuaciones y procesos, pero el poder de decisión, y la orientación planificada en cuanto al manejo de recursos se mantiene radicada y concentrada en el nivel central.

Dicho de otra manera, esto minimiza la oportunidad de redirigir los espacios complementarios de descentralización y participación ciudadana, como elementos de construcción de una paz estable y duradera, que de paso para lograr los objetivos establecidos en la Constitución Política de 1991, donde proclama el acercamiento de los 
ciudadanos al Estado, promueve la participación ciudadana en las decisiones locales, hace la gestión más transparente, y conlleva a mejorar la calidad y la eficiencia en los servicios.

Por consiguiente, se necesitan verdaderas reformas estructurales para fortalecer y modernizar las actuales entidades territoriales, máxime en estos tiempos de transición social y definiciones en materia de paz y de cara a un verdadero posconflicto, cuando es importante afianzar los niveles de decisión de tales niveles geográficos y romper ese modelo centralista asfixiante, que poco ha contribuido a la equidad y al desarrollo integral de algunas zonas del país, dicho de otra manera, hay que impulsar los entes territoriales en la construcción de verdaderos modelos educativos que brinden soluciones al contexto, para entender las realidades cada día más complejas y propiciar caminos de actuación propia y la construcción de espacios geográficos autónomos.

Como resultado a lo anterior, se reduce la capacidad de atención a una educación de calidad, que debe garantizar oportunidades similares a niños y jóvenes de condiciones económicas, sociales, sexuales y raciales diferentes, por lo que a causa de esta situación se puede elevar en el municipio el nivel de inequidad educativa que subsiste en nuestro país.

Finalmente, la descentralización educativa en el municipio de Tunja, desde la autonomía que se le otorga como municipio certificado, se observa como un proceso complejo en tiempo de postconflicto, donde se deben resolver problemas como la retención y permanencia de los estudiantes tanto propios como de todos aquellos que buscan espacios de paz y reconciliación.

\section{Conclusiones}

La descentralización se manifiesta en contextos históricos y geográficos concretos. Colombia, a lo largo de su evolución republicana ha experimentado cambios importantes referidos a su marco referencial. El concepto mismo de descentralización ha sido 
asumido recientemente, con la aprobación de la Constitución Política en el año 1991, momento a partir del cual Colombia asume un nuevo modelo en concordancia con acuerdos internacionales. Esto tiene un gran impacto sobre la organización de las instituciones del Estado y en sus relaciones con los ciudadanos. Estas nuevas exigencias requieren trascender viejos comportamientos, costumbres, aspectos culturales y de idiosincrasia, anclados a paradigmas centralistas de relaciones de poder del siglo XIX y $\mathrm{XX}$, bajo concepciones caudillistas o paternalistas, que aún a pesar de los avances, permanecen en la sociedad y limitan el actuar de los actores del Estado.

Una vez realizado un proceso reflexivo del proceso de descentralización educativa en el Municipio Certificado de Tunja, como un proceso complejo en tiempo de postconflicto, surgen algunas consideraciones, las cuales permitirán, dar aportes significativos al campo investigativo y propiciar nuevas investigaciones que permitan la revisión del contexto social y generen la producción del conocimiento crítico y pertinente en materia de seguridad ciudadana.

La investigación ha permitido el desarrollo de un pensamiento crítico-reflexivo, donde los elementos constitutivos de la descentralización educativa, son comprendidos como fenómenos diversos y multidimensionales, capaces de desarrollar habilidades de adaptación, relación e interconexión con la realidad social.

La revisión de la descentralización educativa del municipio Tunja, desde una visión compleja, genera conocimiento pertinente a la sociedad, donde las disciplinas confluyan y trasciendan el campo social, político, cultural y económico para asumirse como un sistema dentro de otro sistema.

Las relaciones de los elementos que componen el Estado, son complementarias, concurrentes y antagónicas, que permiten un bucle constructivo de conocimiento, donde no solo existe la ciencia y los órganos que la regulan, sino que existen situaciones humanas que implican subjetividad y afectividad. 
Es imperativo, adaptarse a las nuevas conceptualizaciones de democracia, las cuales enfatizan las condiciones para el libre ejercicio de los derechos y la ciudadanía, el desarrollo integral de la sociedad, el logro de la convivencia pacífica y solidaria, la participación, la inclusión social y la gobernabilidad democrática, para lograr la autoproducción, auto organización, auto eco organización, la visión sistémica y la interconexión de los procesos formales de la descentralización.

Impulsar políticas autonomía educativa que propendan a recomponer el tejido social, parte del núcleo familiar y los espacios educativos que da cuenta del individuo en su forma de ser, pensar y actuar con los demás y pone en relieve la experiencia y la conciencia como medio de lograr el desarrollo humano. En este sentido, es necesario hacer énfasis en la idea de que la política educativa desde lo local, requiere el estudio de la vida e historia de los pueblos en relación con su territorio geográfico, para de esta forma pensar acorde a los nuevos tiempos, que favorezca el desarrollo de una cultura de paz y apuntale el desarrollo social, político y cultural.

\section{Bibliografía}

Delgadillo, G. (1989). Descentralización administrativa, desconcentración y delegación de funciones. Tesis para optar al Título de Especialista en Derecho Público. Universidad Nacional de Colombia, Santa Fe de Bogotá, Colombia.

Gómez Aranguren, G. (2002). Principios del derecho Adminsitrativo. ABC Editores, Librería Limitada. Bogotá.

Maldonado, C. E. (2009). La complejidad es un problema, no una cosmovisión. UCM Revista de Investigación, 42-54 p.

Morin, E. (1988). El Método III. El conocimiento del conocimiento. Madrid, España: Cátedra.

Morin, E. (1993). El método II. La vida de la vida. Cátedra. 
Morin, E. (1994). Introducción al pensamiento complejo. Barcelona, España: Gedisa.

Morin, E. (1996): Por una reforma del pensamiento. En: Correo de la UNESCO.

Morin, E. (1999a). La cabeza bien puesta. Repensar la reforma. Reformar el pensamiento. Buenos Aires, Argentina: Ediciones Nueva Visión.

Morin, E. (1999b). Los siete saberes necesarios para la educación del futuro. París, Francia: UNESCO.

Morin, E. (2001). El Método I. La naturaleza de la naturaleza. Cátedra.

Morin, E. (2002). Sociología. Madrid: Tecnos.

Morin, E. (2003). El método 5. La humanidad de la humanidad. La identidad humana. Madrid, España: Ediciones Cátedra.

Morin, E. (2004). Introducción al Pensamiento Complejo (7ma. ed.). México: Gedisa.

Payares, L. (2011). Complejidad y humanidades: conciliando el ser y el devenir. Cuadernos del Centro de Estudios del Desarrollo (CENDES). Venezuela.

Rettberg A. (2015). Academia y reconciliación, en Reconciliación el gran desafío de Colombia Nomos impresores, p. 292

Sánchez, A. (2016). Revisión histórica del proceso de descentralización administrativa y sus retos en Colombia, de 1985 a 2016. Trabajo de grado para optar por el título de Magister en Planeación para el Desarrollo. Universidad Santo Tomás. Colombia.

Tobón, S. Formación basada en competencia. Pensamiento Complejo, diseño curricular y didáctica. $2^{\circ}$ Edición, Bogotá, ECOE Ediciones. (2005). 